\title{
On the distribution of the clipping median under a mixture model
}

\author{
Ansgar Steland ${ }^{1}$ \\ Fakultät für Mathematik \\ Ruhr-Universität Bochum, Germany \\ ansgar.steland@ruhr-uni-bochum.de
}

\begin{abstract}
Motivated by applications in image processing, quality control, and econometrics we derive the exact distribution function of the clipping median estimator which is designed to provide simultaneously robust smooths and jump-preserving reconstructions. We allow for a mixture model which is of special interest for applications in pixel-wise object detection. To construct statistical tests for pixel classification, we propose to rely on estimated $p$-values. Simulations suggest that the resulting approximations are reliable.
\end{abstract}

Keywords: Change point, econometrics, nonparametric regression, image processing, jump-preserving estimation, edge detection, robust statistics.

\section{INTRODUCTION}

Suppose we are given $n$ real-valued random variables (r.v.s) $Y_{1}, \ldots, Y_{n}$ and aim at testing whether $m_{n}=E\left(Y_{n}\right)$ is affected by a level shift when compared to $m_{1}, \ldots, m_{n-1}$, where $m_{i}=E\left(Y_{i}\right), i=1, \ldots, n$. This problem arises in various contexts, e.g., when a sequential monitoring scheme for an economic time series or a series of quality measurements is established for the first time and one wants to compare the current observation $Y_{n}$ with past data $Y_{1}, \ldots, Y_{n-1}$ to detect a level shift with no delay. Such a comparison can also be an useful additional tool for a fixed sample analysis, if the analysis is naively applied

Date: October, 2004.

${ }^{1}$ Address of correspondence: Ansgar Steland, Ruhr-Universität Bochum, Fakultät für Mathematik, Mathematik 3 NA 3/71, Universitätsstr. 150, D-44780 Bochum, Germany. 
in a sequential way after getting the new observation $Y_{n}$, as it is often done in empirical econometric research. In image processing an important task is to decide whether a pixel belongs to the background or to the foreground (object). In this case we may define a local neighborhood of $h=n-1$ neighboring pixels, denote their grey values by $Y_{1}, \ldots, Y_{n-1}$, and add the grey value $Y_{n}$ of the current pixel to the sample. In this article we focus on this image processing problem, last but not least because the estimator has its origins in that field, but our results can be applied in various settings.

Quite often primary interest is in detecting level jumps. An estimator which is especially designed to reproduce (grey level) jumps defining edges and contour lines of objects and their location with high precision is the clipping median of $m_{n}$ defined as the empirical median of all $Y_{i}$ 's with similar values as $Y_{n}$. Here we consider a slightly more general version. Let $k \geq 0$ be a symmetric function $\mathbb{R} \rightarrow \mathbb{R}^{+}$, usually but not necessarily unimodal and decreasing, and $M>0$ a parameter. Common choices for $k$ are the uniform kernel or the Gaussian kernel. Define the clipping median estimator by

$$
\widehat{m}_{n}=\operatorname{ClipMed}\left\{k\left(\left[Y_{i}-Y_{n}\right] / M\right) Y_{i}\right\} \text {, }
$$

where ClipMed is defined as

$$
\operatorname{Med}\left\{k\left(\left[Y_{i}-Y_{n}\right] / M\right) Y_{i}: 1 \leq i \leq n \text { with }\left|Y_{i}-Y_{n}\right| \leq M\right\}
$$

We use the common definition

$$
\operatorname{Med}\left\{\xi_{1}, \ldots, \xi_{n}\right\}= \begin{cases}\frac{1}{2}\left(\xi_{(n / 2+1)}+\xi_{(n / 2)}\right), & n \text { even } \\ \xi_{([n+1] / 2)}, & n \text { odd }\end{cases}
$$

of the empirical median of $n$ r.v.s $\xi_{1}, \ldots, \xi_{n}$, where $\xi_{(1)} \leq \cdots \leq \xi_{(n)}$ denotes the corresponding order statistic. Recall that the exact distribution for i.i.d. observations is related to the binomial distribution due to the relationship

$$
\operatorname{Med}\left\{\xi_{1}, \ldots, \xi_{n}\right\} \leq x \Leftrightarrow \sum_{i=1}^{n} \mathbf{1}\left(\xi_{i} \geq x\right) \leq(n+1) / 2
$$

which holds true for both even and odd $n$. For the clipping median the situation is more involved and is the topic of this paper.

The particular version (1) has been studied in Pawlak, Rafajłowicz and Steland (2004), where sufficient conditions were derived which ensure that the sequential stopping rule $\inf \left\{n \in \mathbb{N}: \widehat{m}_{n}>c\right\}$ can detect jumps in time series with no delay, if $c$ is appropriately 
chosen. Note that $\widehat{m}_{n}$ reduces to the empirical median of all $Y_{i}$ such that $\left|Y_{i}-Y_{n}\right| \leq M$ if $k$ is the uniform kernel. However, the behavior of $\widehat{m}_{n}$ for general $k$ is as follows. Observations with $\left|Y_{i}-Y_{n}\right|>M$, i.e., far away from the current observation, are excluded from the calculation (clipping). The kernel $k$ performs a shrinkage operation, since observations $Y_{i}$ with large values of $\left|Y_{i}-Y_{n}\right|$, which are not ignored by the clipping mechanism, are shrunken towards 0 . This means, the data transformation $Y_{i} \mapsto Z_{i}=k\left(\left[Y_{i}-Y_{n}\right] / M\right) Y_{i}$ forms a cluster. The clipping mechanism ensures that the sample from which the median is calculated shrinks substantially, if the current observation is different from the other ones. This property ensures that sufficiently large level shifts can be reproduced more accurately than with classic averaging procedures. If $\left|Y_{i}-Y_{n}\right|>M$ for all $1 \leq i<n$, the clipping median interpolates, i.e., $\widehat{m}_{n}=Y_{n}$. Finally note that if the sample is homogenous (i.i.d.) with median 0 , we have $\operatorname{Med} k\left(\left[Y_{i}-Y_{n}\right] / M\right) Y_{i}=0,1 \leq i \leq n$. If there is a level shift, the $Z_{i}$ s before the shift will be shrunken to 0 if $k$ is chosen appropriately, whereas the $Z_{i}$ s afer the shift are not shrunken.

The basic idea underlying the estimator $\widehat{m}_{n}$ can be traced back to Lee (1983) who studied the problem of edge-preserving estimators for image processing purposes. Lee's estimator is implemented in many image processing packages including Mathematica, see WolframResearch (2004). It has been studied in different contexts, e.g., to smooth magnetic resonance (MR) images. That application has been studied by Godtliebsen (1991), Godtliebsen and Spøtvoll (1991), chapter 4 of Budinger et al. (1996), and Chiu et al. (1998). The latter paper also discusses the relationship to $M$ estimation. For a discussion of an application of nonlinear Gaussian filters to images we refer to Godtliebsen and Marron (1997). Further recent work can be found in Pawlak and Rafajłowicz (2001). The application of jump-preserving estimators for sequential monitoring and related theoretical results can be found in Pawlak and Rafajłowicz (2000), Steland (2002a, 2002b, 2004a), and Pawlak, Rafajłowicz and Steland (2004). For related recent results on classic kernel estimators we refer to Steland (2004b) and the references given there.

The contribution of this article is to provide a theoretical basis for deriving statistical test procedures based on the clipping median. We consider two models. In Section 1 we study the d.f. of $\widehat{m}_{n}$ assuming that the neighborhood $Y_{1}, \ldots, Y_{n-1}$ forms a homogenous i.i.d. sample. Section 2 considers a more general mixture model. Finally, in Section 3 we study the a.s. convergence of the proposed estimated $p$ values. 


\section{I.I.D. NeIGHBORHOOdS}

Let us first study a setting which can be used to decide whether or not a single pixel belongs to a homogeneous background. Assume $Y_{1}, \ldots, Y_{n}$ are independent such that $Y_{1}, \ldots, Y_{n-1}$ are i.i.d. with common d.f. $F(x)$, and $Y_{n} \sim F(x-\Delta)$ for some shift $\Delta \in \mathbb{R}$. We aim at testing $H_{0}: \Delta=0$ versus $H_{1}: \Delta \neq 0$ based on the test statistic $\widehat{m}_{n}$. Let

$$
G_{n}(x ; \Delta)=P_{\Delta}\left(\widehat{m}_{n} \leq x\right), \quad x \in \mathbb{R},
$$

denote the d.f. of the clipping median estimator $\widehat{m}_{n}$.

Further, let $p_{1}, p_{2} \in[0,1]$ be two probabilities and denote by

$$
q\left(n, i, k, p_{1}, p_{2}\right)=\frac{n !}{i ! k !(n-i-k) !} p_{1}^{i} p_{2}^{k}\left[1-p_{1}-p_{2}\right]^{n-i-k}
$$

the trinomial probabilites, where $n \in \mathbb{N}, i, k \geq 0$, and $i+k \leq n$. We set $q(n, i, k)=$ 0 whenever the constraints on the indices are not satisfied. For an event $A$ (or logical expression) $\mathbf{1}(A)$ is 1 if $A$ occurs (is true), and equals 0 otherwise.

Theorem 1. Let $Y_{1}, \ldots, Y_{n}$ be independent random variables such that $Y_{1}, \ldots, Y_{n-1}$ are i.i.d. with c.d.f. $F$ and $Y_{n} \sim F(x-\Delta)$ for some shift $\Delta \in \mathbb{R}$. For all $x \in \mathbb{R}$ we have

$$
G_{n}(x ; \Delta)=\int \sum_{(k, i) \in \mathcal{J}(x, y)} q\left(n-1, k, i, p_{+}(x, y), p_{-}(x, y)\right) d F(y-\Delta),
$$

where $\mathcal{J}=\left\{(k, i) \in\{0, \ldots, n-1\}^{2}: k-i \leq 2 \mathbf{1}(y>k(0) / M)\right\}$ and

$$
\begin{aligned}
& p_{+}(x, y)=P\left(\left|Y_{1}-y\right| \leq M, k\left(\left[Y_{1}-y\right] / M\right) Y_{1} \geq x\right), \\
& p_{-}(x, y)=P\left(\left|Y_{1}-y\right| \leq M, k\left(\left[Y_{1}-y\right] / M\right) Y_{1}<x\right),
\end{aligned}
$$

for $x, y \in \mathbb{R}$.

Proof. We shall consider the case $\Delta=0$, the general case $\Delta \in \mathbb{R}$ will be obvious. Hence, we have for each $x \in \mathbb{R}$

$$
G_{n}(x ; 0)=\int P\left[\operatorname{Med}\left\{k\left(\left[Y_{i}-y\right] / M\right) Y_{i}:\left|Y_{i}-y\right| \leq M\right\} \leq x \mid Y_{n}=y\right] d F(y) .
$$

To calculate the integrand let

$$
\mathcal{L}(y)=\left\{i \in\{1, \ldots, n\}:\left|Y_{i}-y\right| \leq M\right\}
$$


be the random set of all indices corresponding to the clipped observations and put $L(y)=$ $|\mathcal{L}(y)|$. Note that $n \in \mathcal{L}(y)$. Recall that by definition of the clipping median,

$$
\text { ClipMed }\left\{Z_{i}(y)\right\} \leq x \quad \Leftrightarrow \quad S_{L(y)}(x) \leq[L(y)+1] / 2,
$$

for both $L(y)$ odd and even, where

$$
S_{l}=\sum_{i=1}^{l} \mathbf{1}\left(Z_{i}(y) \geq x\right), \quad l \in \mathbb{N},
$$

with $Z_{i}(y)=k\left(\left[Y_{i}-y\right] / M\right) Y_{i}, i=1, \ldots, n$. Consequently,

$$
G_{n}(x ; 0)=\int P\left[S_{L(y)}(x)-(L(y)+1) / 2 \leq 0 \mid Y_{n}=y\right] d F(y) .
$$

We have

$$
\begin{aligned}
L(y) & =\sum_{i=1}^{n-1} \mathbf{1}\left(\left|Y_{i}-y\right| \leq M\right)+1 \\
S_{L(y)}(x) & =\sum_{i \in \mathcal{L}(y)} \mathbf{1}\left(Z_{i}(y) \geq x\right) \\
& =\sum_{i=1}^{n-1} \mathbf{1}\left(\left|Y_{i}-y\right| \leq M\right) \mathbf{1}\left(Z_{i}(y) \geq x\right)+\mathbf{1}(y \geq x / k(0))
\end{aligned}
$$

Thus, $S_{L(y)}(x)-L(y) / 2 \leq 0$ is equivalent to

$$
\sum_{i=1}^{n-1} \eta_{i}(x, y) \leq 2 \mathbf{1}(y>k(0) / M)
$$

where

$$
\eta_{i}(x, y)=2 \mathbf{1}\left(\left|Y_{i}-y\right| \leq M\right)\left\{\mathbf{1}\left(Z_{i}(y) \geq x\right)-1 / 2\right\}, \quad i=1, \ldots, n-1,
$$

are i.i.d. $\{-1,0,+1\}$-valued r.v.s with

$$
P\left(\eta_{1}(x, y)=k \mid Y_{n}=y\right)=p_{-}(x, y)^{\mathbf{1}(k=-1)} p_{+}(x, y)^{\mathbf{1}(k=1)}\left[1-p_{+}(x, y)-p_{-}(x, y)\right]^{\mathbf{1}(k=0)} .
$$

By independence of $\eta_{i}(x, y), i=1, \ldots, n$, the random vector $\left(N_{+}(x, y), N_{-}(x, y)\right)$, where

$$
\begin{aligned}
& N_{+}(x, y)=\sum_{i=1}^{n-1} \mathbf{1}\left(\delta_{i}(x, y)=+1\right), \\
& N_{-}(x, y)=\sum_{i=1}^{n-1} \mathbf{1}\left(\delta_{i}(x, y)=-1\right),
\end{aligned}
$$


follows a trinomial distribution with parameters $n-1, p_{+}(x, y)$, and $p_{-}(x, y)$, Therefore, we obtain

$$
\begin{aligned}
P & {\left[S_{L(y)}-(L(y)+1) / 2 \leq 0 \mid Y_{n}=y\right] } \\
& =P\left[N_{+}(x, y)-N_{-}(x, y) \leq 2 \mathbf{1}(y>k(0) / M) \mid Y_{n}=y\right] \\
& =\sum_{(k, i) \in \mathcal{J}(x, y)} q\left(n-1, k, i, p_{+}(x, y), p_{-}(x, y)\right) .
\end{aligned}
$$

Obviously, the last expression is integrable w.r.t. $d F(y)$. Hence,

$$
G_{n}(x ; \Delta)=\int \sum_{(k, i) \in \mathcal{J}(x, y)} q\left(n-1, k, i, p_{+}(x, y), p_{-}(x, y)\right) d F(y)
$$

If $Y_{n} \sim F(x-\Delta)$, the measure $d F(y)$ is replaced by $d F(y-\Delta)$.

This result suggests the following estimators for the d.f. of the clipping median under both the null hypothesis and the alternative. For $\Delta=0$ let

$$
\begin{aligned}
\widehat{G}_{L_{1}, L_{2}, n}(x ; 0) & =\int \sum_{(k, i) \in \mathcal{J}^{*}(x, y)} q\left(n-1, k, i, p_{+}(x, y), p_{-}(x, y) d \widehat{F}_{n}(y)\right. \\
& =\frac{1}{n-1} \sum_{(k, i) \in \mathcal{J}^{*}\left(x, Y_{j}\right)} q\left(n-1, k, i, p_{+}\left(x, Y_{j}\right), p_{-}\left(x, Y_{j}\right),\right.
\end{aligned}
$$

where

$$
\begin{aligned}
& \mathcal{J}^{*}(x, y)=\{(k, i) \in\{0, \ldots, n-1\}^{2}:\left|k-(n-1) p_{+}(x, y)\right| \leq L_{1}, \\
&\left.\left|i-(n-1) p_{-}(x, y)\right| \leq L_{2},|k-i| \leq 2 \mathbf{1}(y>k(0) / M)\right\} .
\end{aligned}
$$

$L_{1}, L_{2}$ are truncation constants selecting the central atoms around the means. $\widehat{F}_{n-1}(x)=$ $(n-1)^{-1} \sum_{j=0}^{n-1} \mathbf{1}\left(Y_{j} \leq x\right)$ is the e.d.f. of $Y_{1}, \ldots, Y_{n-1}$, and

$$
\begin{aligned}
& \widehat{p}_{1}(x, y)=\frac{1}{n-1} \sum_{j=1}^{n-1} \mathbf{1}\left(\left|Y_{n-j}-y\right| \leq M, Z_{n-j}(y) \geq x\right), \\
& \widehat{p}_{2}(x, y)=\frac{1}{n-1} \sum_{j=1}^{n-1} \mathbf{1}\left(\left|Y_{n-j}-y\right| \leq M, Z_{n-j}(y)<x\right) .
\end{aligned}
$$


For $\Delta \neq 0$ the d.f. can be estimated by replacing $d \widehat{F}_{n-1}(y)$ in (3) by $d \widehat{F}_{n-1}(y-\Delta)$. Thus, define $\widehat{G}_{n}(x ; \Delta)$ as

$$
\frac{1}{n-1} \sum_{(k, i) \in \mathcal{J}^{*}\left(x, Y_{j}+\Delta\right)} q\left(n-1, k, i, p_{+}\left(x, Y_{j}+\Delta\right), p_{-}\left(x, Y_{j}+\Delta\right)\right),
$$

where $\widehat{p}_{+}(x, y)$ and $\widehat{p}_{-}(x, y)$ are defined as in (4) and (5), respectively.

We now propose to test $H_{0}: \Delta=0$ against $H_{1}: \Delta \neq 0$ using the test $\phi$ given by

$$
\phi=\mathbf{1}\left(\widehat{p}_{L_{1}, L_{2}, n} \notin(\alpha / 2,1-\alpha / 2)\right)
$$

where

$$
\widehat{p}_{L_{1}, L_{2}, n}=\widehat{G}_{L_{1}, L_{2}, n}\left(\widehat{m}_{n} ; 0\right)
$$

is the estimated $p$ value. The a.s. convergence of the estimated $p$ value will be discussed in Section 3 using the more general framework of the next section.

\section{Mixture Model for Neighborhoods}

We shall now generalize the results of the previous section to a mixture model for the neighborhood of the current observation $Y_{n}$. So let $Y_{1}, \ldots, Y_{n}$ be independent r.v.s. We assume that $k Y_{i}$ s are distributed according to $F(x)$ (background), $n-1-k$ have distribution $F(x-\Delta)$ (object), and $Y_{n} \sim F\left(x-\Delta^{\prime}\right)$. This means, there exists a decomposition $\{1, \ldots, n-$ $1\}=\mathcal{I}_{1}+\mathcal{I}_{2}$ into disjoint nonempty sets $\mathcal{I}_{1} \subset\{1, \ldots, n-1\}$ and $\mathcal{I}_{2} \subset\{1, \ldots, n-1\}$ with $\left|\mathcal{I}_{1}\right|=k$ and $\left|\mathcal{I}_{2}\right|=n-1-k$, such that

$$
\begin{gathered}
Y_{i} \sim F(x), \quad i \in \mathcal{I}_{1}, \\
Y_{i} \sim F(x-\Delta), \quad i \in \mathcal{I}_{2} .
\end{gathered}
$$

Further, assume

$$
Y_{n} \sim F\left(x-\Delta^{\prime}\right)
$$

Here $\Delta, \Delta^{\prime} \in \mathbb{R}$ are level shifts. Provided $\Delta$ is known, the testing problem of interest is $H_{0}: \Delta^{\prime}=\Delta$ versus $H_{1}: \Delta^{\prime}=0$ to reveal that $Y_{n}$ is a background pixel, or $H_{0}: \Delta^{\prime}=0$ versus $H_{1}: \Delta^{\prime}=\Delta$ to reveal that $Y_{n}$ belongs to the object.

Put $\Delta=\left(\Delta, \Delta^{\prime}\right)$ and let

$$
G_{n}(x ; \boldsymbol{\Delta})=P_{\boldsymbol{\Delta}}\left[\widehat{m}_{n} \leq x\right], \quad x \in \mathbb{R},
$$


denote the d.f. of the clipping median, where $P_{\boldsymbol{\Delta}}$ indicates that the probability is calculated assumming the mixture model given by (8), (9), and (10) holds true.

Theorem 2. Let $Y_{1}, \ldots, Y_{n}$ be independent random variables such that (8), (9), and (10) hold true. Let $\mathcal{I}_{1}+\mathcal{I}_{2}=\{1, \ldots, n-1\}$ be a disjoint decomposition in two non-empty sets. For all $x \in R$ we have

$$
G_{n}^{\left(\mathcal{I}_{1}, \mathcal{I}_{2}\right)}(x ; \boldsymbol{\Delta})=\int \sum_{(k, i) \in \mathcal{J}(x, y)} P_{1,\left|\mathcal{I}_{1}\right|}(x, y, k) P_{2 \Delta,\left|\mathcal{I}_{2}\right|}(x, y, i) d F\left(y-\Delta^{\prime}\right),
$$

where $\mathcal{J}(x, y)=\left\{(k, i) \in \mathbb{N}^{2}:|k| \leq\left|\mathcal{I}_{1}\right|,|i| \leq\left|\mathcal{I}_{2}\right|, k+i \leq 2 \mathbf{1}(y>k(0) / M)\right\}$, and

$$
\begin{aligned}
P_{1, l}(x, y, r) & =\sum_{i=-r}^{l-r} q\left(\left|\mathcal{I}_{1}\right|, i, r+i, p_{+}(x, y ; 0), p_{-}(x, y, 0)\right),|r| \leq\left|\mathcal{I}_{1}\right|, \\
P_{2 \Delta, l}(x, y, r) & =\sum_{i=-r}^{l-r} q\left(\left|\mathcal{I}_{2}\right|, i, r+i, p_{+}(x, y ; \Delta), p_{-}(x, y ; \Delta)\right),|r| \leq\left|\mathcal{I}_{2}\right|,
\end{aligned}
$$

with

$$
\begin{aligned}
& p_{+}(x, y ; \delta)=P(|Y+\delta-y| \leq M, k([Y+\delta-y] / M) Y \geq x), \\
& p_{-}(x, y ; \delta)=P(|Y+\delta-y| \leq M, k([Y+\delta-y] / M) Y<x),
\end{aligned}
$$

for $\delta \in \mathbb{R}$.

Proof. The proof is similar as the proof of Theorem 1. Let $\widetilde{Y}_{1}, \ldots, \widetilde{Y}_{n} \stackrel{i . i . d .}{\sim} F(x)$. Then we may assume $Y_{i}=\widetilde{Y}_{i}$ if $i \in \mathcal{I}_{1}, Y_{i}=\widetilde{Y}_{i}+\Delta$ if $i \in \mathcal{I}_{2}$, and $Y_{n}=\widetilde{Y}_{n}+\Delta^{\prime}$. Using the same notation as in the proof of Theorem 1 , we have given $Y_{n}=y$

$$
\begin{aligned}
L(y ; \Delta)= & \sum_{i \in \mathcal{I}_{1}} \mathbf{1}\left(\left|\widetilde{Y}_{i}-y\right| \leq M\right)+\sum_{i \in \mathcal{I}_{2}} \mathbf{1}\left(\left|\widetilde{Y}_{i}+\Delta-y\right| \leq M\right)+1 \\
S_{L(y)}(x ; \Delta)= & \sum_{i \in \mathcal{I}_{1}} \mathbf{1}\left(\left|\widetilde{Y}_{i}-y\right| \leq M\right) \mathbf{1}\left(\widetilde{Z}_{i}(y ; 0) \geq x\right) \\
& \quad+\sum_{i \in \mathcal{I}_{2}} \mathbf{1}\left(\left|\widetilde{Y}_{i}+\Delta-y\right| \leq M\right) \mathbf{1}\left(\widetilde{Z}_{i}(y ; \Delta) \geq x\right)+\mathbf{1}(y \geq x / k(0))
\end{aligned}
$$

where

$$
\widetilde{Z}_{i}(y ; \delta)=\mathbf{1}\left(k\left(\left[\widetilde{Y}_{i}+\delta-y\right] / M\right)\left(\widetilde{Y}_{i}+\delta\right),\right.
$$

$i=1, \ldots, n$. Now $S_{L(y)}(x ; \Delta)-L(y ; \Delta) / 2 \leq 0$ is equivalent to

$$
C(x, y ; \Delta) \leq \underset{8}{2 \mathbf{1}}(y \geq k(0) / M),
$$


where

$$
C(x, y ; \Delta)=S_{1}(x, y)+S_{2}(x, y ; \Delta)
$$

with

$$
S_{1}(x, y)=\sum_{i \in \mathcal{I}_{1}} \eta_{i}(x, y ; 0), \quad S_{2}(x, y ; \Delta)=\sum_{i \in \mathcal{I}_{2}} \eta_{i}(x, y ; \Delta)
$$

and

$$
\eta_{i}(x, y ; \delta)=2 \mathbf{1}\left(\left|\widetilde{Y}_{i}+\delta-y\right| \leq M\right)\left\{\mathbf{1}\left(\widetilde{Z}_{i}(y ; \delta) \geq x\right)-1 / 2\right\},
$$

$i=1, \ldots, n-1$. Clearly,

$$
S_{1}(x, y) \sim P_{1}(x, y ; \circ) \quad \text { and } \quad S_{2}(x, y ; \Delta) \sim P_{2 \Delta}(x, y ; \circ)
$$

where the probability functions $P_{1}(x, y ; \circ)$ and $P_{2 \Delta}(x, y ; \circ)$ are defined in (11) and (12). By independence of $S_{1}(x, y)$ and $S_{2}(x, y ; \Delta)$, we have

$$
P\left(S_{1}(x, y)+S_{2}(x, y) \leq 2 \mathbf{1}(y>k(0) / M) \mid Y_{n}=y\right)=\sum_{(k, i) \in \mathcal{J}(x, y)} P_{1,\left|\mathcal{I}_{1}\right|}(x, y, k) P_{2 \Delta,\left|\mathcal{I}_{2}\right|}(x, y, i) .
$$

Therefore, we obtain

$$
G_{n}^{\left(\mathcal{I}_{1}, \mathcal{I}_{2}\right)}(x ; \boldsymbol{\Delta})=\int \sum_{(k, i) \in \mathcal{J}(x, y)} P_{1,\left|\mathcal{I}_{1}\right|}(x, y, k) P_{2 \Delta,\left|\mathcal{I}_{2}\right|}(x, y, i) d F\left(y-\Delta^{\prime}\right),
$$

The definition of appropriate estimators strongly depends on the application. For instance, for certain applications in image processing one would prefer to estimate background and/or foreground using external data. If no external data is available or a local estimation procedure seems to be more appropriate one can proceed as follows. Calculate residuals

$$
\begin{aligned}
& \widehat{\epsilon}_{i}=Y_{i}, \quad i \in \mathcal{I}_{1}, \\
& \widehat{\epsilon}_{i}=Y_{i}-\widehat{\Delta}, \quad i \in \mathcal{I}_{2},
\end{aligned}
$$

where, e.g, $\widehat{\Delta}=\left|\mathcal{I}_{2}\right|^{-1} \sum_{i \in \mathcal{I}_{2}} Y_{i}$. Let $\widehat{F}_{n}(x)=(n-1)^{-1} \sum_{i=1}^{n-1} \mathbf{1}\left(\widehat{\epsilon}_{i} \leq x\right)$. The d.f. of the clipping median under the mixture model can now be estimated by

$$
\widehat{G}_{L_{1}, L_{2}, n}^{\left(\mathcal{I}_{1}, \mathcal{I}_{2}\right)}(x ; \boldsymbol{\Delta})=\int \sum_{(k, i) \in \mathcal{J}^{*}(x, y)} \widehat{P}_{1, L_{1}}(x, y, k) \widehat{P}_{2 \Delta, L_{2}}(x, y, i) d \widehat{F}_{n}\left(y-\Delta^{\prime}\right)
$$


where $\mathcal{J}^{*}(x, y)=\left\{(k, i) \in \mathbb{N}^{2}:\left|k-(n-1) p_{+}(x, y)\right| \leq L_{1},\left|i-(n-1) p_{-}(x, y)\right| \leq L_{2}, k+i \leq\right.$ $2 \mathbf{1}(y>k(0) / M)\}$ for truncation constants $L_{1}$ and $L_{2}$. Further,

$$
\begin{aligned}
\widehat{P}_{1, L_{1}}(x, y, r) & =\sum_{i=-r}^{L_{1}-r} q\left(\left|\mathcal{I}_{1}\right|, i, r+i, \widehat{p}_{+n}(x, y ; 0), \widehat{p}_{-n}(x, y, 0)\right),|r| \leq\left|\mathcal{I}_{1}\right|, \\
\widehat{P}_{2 \Delta, L_{2}}(x, y, r) & =\sum_{i=-r}^{L_{2}-r} q\left(\left|\mathcal{I}_{2}\right|, i, r+i, \widehat{p}_{+n}(x, y ; \Delta), \widehat{p}_{-n}(x, y ; \Delta)\right),|r| \leq\left|\mathcal{I}_{2}\right|,
\end{aligned}
$$

with

$$
\begin{aligned}
& \widehat{p}_{+n}(x, y ; \delta)=(n-1)^{-1} \sum_{i=1}^{n-1} \mathbf{1}\left(\left|\widehat{\epsilon}_{i}+\delta-y\right| \leq M, k\left(\left[\widehat{\epsilon}_{i}+\delta-y\right] / M\right) \widehat{\epsilon}_{i} \geq x\right), \\
& \widehat{p}_{-n}(x, y ; \delta)=(n-1)^{-1} \sum_{i=1}^{n-1} \mathbf{1}\left(\left|\widehat{\epsilon}_{i}+\delta-y\right| \leq M, k\left(\left[\widehat{\epsilon}_{i}+\delta-y\right] / M\right) \widehat{\epsilon}_{i}<x\right),
\end{aligned}
$$

for $\delta \in \mathbb{R}$.

Now we may test $H_{0}: \Delta^{\prime}=\Delta$ versus $H_{1}: \Delta^{\prime} \neq \Delta$ using the test

$$
\phi_{n}=\mathbf{1}\left(\widehat{p}_{L_{1}, L_{2}, n} \notin[\alpha / 2,1-\alpha / 2]\right) .
$$

where

$$
\widehat{p}_{L_{1}, L_{2}, n}=\widehat{G}_{L_{1}, L_{2}, n}^{\left(\mathcal{I}_{1}, \mathcal{I}_{2}\right)}\left(\widehat{m}_{n} ; \widehat{\boldsymbol{\Delta}}\right)
$$

is the estimated $p$-value.

\section{Convergence of $p$-Values}

It remains to discuss the convergence of the proposed estimators for the $p$ values associated to the testing problem. We consider the general mixture model case studied in the previous section. The following theorem provides sufficient conditions for a.s. convergence of the distance between $\widehat{p}_{L_{1}, L_{2}, n}$ and the truncated $p$-value

$$
G_{L_{1}, L_{2}, n}^{\left(\mathcal{I}_{1}, \mathcal{I}_{2}\right)}\left(\widehat{m}_{n} ; \Delta, \Delta\right)=\int \sum_{(k, i) \in \mathcal{J}^{*}\left(\widehat{m}_{n}, y\right)} P_{1, L_{1}}\left(\widehat{m}_{n}, y, k\right) P_{2 \Delta, L_{2}}\left(\widehat{m}_{n}, y, i\right) d F(y-\Delta) .
$$

For sufficiently large $L_{1}$ and $L_{2}$ the distance to the true $p$-value $G_{n}^{\left(\mathcal{I}_{1}, \mathcal{I}_{2}\right)}\left(\widehat{m}_{n} ; \Delta, \Delta\right)$ is small.

Theorem 3. Let $F$ be a Lipschitz continuous d.f. Assume (8), (9), (10), and

$$
0<c_{1} \leq \frac{\left|\mathcal{I}_{1}\right|}{n}, \frac{\left|\mathcal{I}_{2}\right|}{n} \leq c_{2}<1
$$


for constants $c_{1}, c_{2}$. Suppose

$$
\widehat{\Delta}_{n} \stackrel{a . s .}{\rightarrow} \Delta, \quad n \rightarrow \infty
$$

and

(KC) The kernel $k$ is continuous and the sets $\{\widetilde{y}: k([\widetilde{y}-(y-\delta)] / M) \widetilde{y} \geq x\}$ are intervals $[A(x, y, \delta), B(x, y, \delta)]$ with continuous functions $A$ and $B$.

Then

$$
\sup _{x}\left|\widehat{G}_{L_{1}, L_{2}, n}^{\left(\mathcal{I}_{1}, \mathcal{I}_{2}\right)}\left(x ;\left(\widehat{\Delta}_{n}, \widehat{\Delta}_{n}\right)\right)-G_{L_{1}, L_{2}, n}^{\left(\mathcal{I}_{1}, \mathcal{I}_{2}\right)}\left(x ;\left(\widehat{\Delta}_{n}, \widehat{\Delta}_{n}\right)\right)\right| \stackrel{P}{\rightarrow} 0,
$$

as $n \rightarrow \infty$.

Remark 3.1. Condition $(K C)$ is satisfied by many kernels, e.g, the Gaussian kernel for which $j(z)=z K([z-a] / M), a \in \mathbb{R}$, is unimodal and concave. The implicit function theorem ensures that the solutions $z=z(c, a, M)$ of $j(z)=x, x>0$, depend continuously on $c, a$, and $M$. Thus $A$ and $B$ are continuous.

Proof. Clearly, $\widehat{\Delta}_{n} \stackrel{\text { a.s. }}{\rightarrow} \Delta, n \rightarrow \infty$, implies that

$$
\max _{i=1, \ldots, n}\left|\widehat{\epsilon}_{i}-\epsilon_{i}\right| \stackrel{a . s .}{\rightarrow} 0
$$

as $n \rightarrow \infty$, where $\epsilon_{i}=Y_{i}$ if $i \in \mathcal{I}_{1}, \epsilon_{i}=Y_{i}-\Delta$, if $i \in \mathcal{I}_{2}, \widehat{\epsilon}_{i}=Y_{i}, i \in \mathcal{I}_{1}$, and $\widehat{\epsilon}_{i}=Y_{i}-\widehat{\Delta}_{n}$, $i \in \mathcal{I}_{2}$. Therefore the e.d.f. of $\widehat{\epsilon}_{1}, \ldots, \widehat{\epsilon}_{n}$ converges to $F(x)$, uniformly in $x \in \mathbb{R}$. This implies weak convergence, i.e.,

$$
\int g(y) d \widehat{F}_{n}\left(y-\Delta^{\prime}\right) \rightarrow \int g(y) d F\left(y-\Delta^{\prime}\right)
$$

a.s., as $n \rightarrow \infty$, for all bounded and continuous functions $g$. Note that for $\mathbb{R}$-valued functions $g_{n}, g$ defined on $\mathbb{R}^{3}$ the estimate

$$
\begin{aligned}
& \left|\int \widehat{g}_{n}(x, y, \delta) d \widehat{F}_{n}(y)-\int g(x, y, \delta) d F(y)\right| \\
& \quad \leq \sup _{x, y, \delta}\left|\widehat{g}_{n}(x, y, \delta)-g(x, y, \delta)\right|+\int g(x, y, \delta) d\left(\widehat{F}_{n}-F\right)(y)
\end{aligned}
$$

also implies

as $n \rightarrow \infty$, if

$$
\sup _{x, \delta}\left|\int \widehat{g}_{n}(x, y, \delta) d \widehat{F}_{n}(y)-\int g(x, y, \delta) d F(y)\right| \stackrel{a . s .}{\rightarrow} 0
$$

$$
\left\|\widehat{g}_{n}-g\right\|_{\infty} \stackrel{a . s .}{\rightarrow} 0
$$


as $n \rightarrow \infty$, and $\|g\|_{\infty}<\infty$.

Note that

$$
\widehat{G}_{L_{1}, L_{2}, n}^{\left(\mathcal{I}_{1}, \mathcal{I}_{2}\right)}(x ; \widehat{\boldsymbol{\Delta}})=\int \sum_{(k, i) \in \mathcal{J}^{*}\left(x, y+\widehat{\Delta}_{n}\right)} \widehat{P}_{1, L_{1}}\left(x, y+\widehat{\Delta}_{n}\right) \widehat{P}_{2 \widehat{\Delta}_{n}, L_{2}}\left(x, y+\widehat{\Delta}_{n}\right) d F(y) .
$$

Thus, the assertion follows if we verify (19) for the functions

$$
\begin{aligned}
\widehat{g}_{n}(x, y, \delta) & =\sum_{(k, i) \in \mathcal{J}^{*}(x, y+\delta)} \widehat{P}_{1, L_{1}}(x, y+\delta) \widehat{P}_{2 \delta, L_{2}}(x, y+\delta) \\
g(x, y, \delta) & =\sum_{(k, i) \in \mathcal{J}^{*}(x, y+\delta)} P_{1, L_{1}}(x, y+\delta, k) P_{2 \delta, L_{2}}(x, y+\delta, i),
\end{aligned}
$$

since $\|g\|_{\infty} \leq 1$. We start by showing that $\widehat{p}_{+n}(x, y ; \delta) \rightarrow p_{+}(x, y ; \delta)$, as $n \rightarrow \infty$, a.s. Define

$$
\widetilde{p}_{+n}(x, y ; \delta)=\frac{1}{n-1} \sum_{i=1}^{n-1} \mathbf{1}\left(\epsilon_{i} \in[(y-\delta)-M,(y-\delta)+M], k\left(\left[\epsilon_{i}-\delta\right) / M\right] \epsilon_{i} \geq x\right) .
$$

Note that by $(\mathrm{KC})$ there exist functions $I_{1}(x, y, \delta)$ and $I_{2}(x, y, \delta)$ such that

$$
\widetilde{p}_{+n}(x, y ; \delta)=\frac{1}{n-1} \sum_{i=1}^{n-1} \mathbf{1}\left(\epsilon_{i} \in\left[I_{1}(x, y, \delta), I_{2}(x, y, \delta)\right]\right) .
$$

Thus, by uniformity of the Glivenko-Cantelli Theorem over VC classes (see Blum (1955), DeHardt (1971), and Shorack and Wellner (1986, Ch. 26, Sec. 1, Th. 1)) we obtain

$$
\sup _{x, y, \delta}\left|\widetilde{p}_{+n}(x, y, \delta)-p_{+}(x, y, \delta)\right| \stackrel{a . s .}{\rightarrow} 0,
$$

as $n \rightarrow \infty$. Note that

$$
\left|\widehat{p}_{+n}(x, y, \delta)-\widetilde{p}_{+n}(x, y, \delta)\right|=(n-1)^{-1} \sum_{i=1}^{n-1}\left[\xi_{i}^{\prime}(x, y, \delta)+\eta_{i}^{\prime}(x, y, \delta)\right]
$$

where

$$
\begin{aligned}
& \xi_{i}^{\prime}(x, y, \delta)=\mathbf{1}\left(\epsilon_{i} \notin\left[I_{1}(x, y, \delta), I_{2}(x, y, \delta)\right], \widehat{\epsilon}_{i} \in\left[I_{1}(x, y, \delta), I_{2}(x, y, \delta)\right]\right), \\
& \zeta_{i}^{\prime}(x, y, \delta)=\mathbf{1}\left(\epsilon_{i} \in\left[I_{1}(x, y, \delta), I_{2}(x, y, \delta)\right], \widehat{\epsilon}_{i} \notin\left[I_{1}(x, y, \delta), I_{2}(x, y, \delta)\right]\right),
\end{aligned}
$$

$i=1, \ldots, n$. Let $\eta>0$. On a set $Z$ with $P(Z)=1$ there exists a $n_{0} \in \mathbb{N}$ with $E_{n}=$ $\max _{1 \leq i \leq n}\left|\widehat{\epsilon}_{i}-\epsilon_{i}\right|<\eta$ for all $n \geq n_{0}$. Writing $\widehat{\epsilon}_{i}=\epsilon_{i}+\left(\widehat{\epsilon}_{i}-\epsilon_{i}\right)$ we may estimate

$$
\begin{aligned}
\xi_{i}^{\prime}(x, y, \delta) & \leq \mathbf{1}\left(\epsilon_{i} \notin\left[I_{1}(x, y, \delta), I_{2}(x, y, \delta)\right], \epsilon_{i} \in\left[I_{1}(x, y, \delta)-\eta, I_{2}(x, y, \delta)+\eta\right]\right) \\
& \leq \xi_{i}(x, y, \delta)=\mathbf{1}\left(\epsilon_{i} \in\left[I_{1}(x, y, \delta)-\eta, I_{1}(x, y, \delta)\right] \cup\left[I_{2}(x, y, \delta), I_{2}(x, y, \delta)+\eta\right]\right)
\end{aligned}
$$


and, similarly,

$$
\zeta_{i}^{\prime}(x, y, \delta) \leq \zeta_{i}(x, y, \delta)=\mathbf{1}\left(\epsilon_{i} \in\left[I_{1}(x, y, \delta), I_{1}(x, y, \delta)+\eta\right] \cup\left[I_{2}(x, y, \delta)-\eta, I_{2}(x, y, \delta)\right] .\right.
$$

Again, since the Glivenko-Cantelli Theorem holds uniformly over VC classes,

$$
\sup _{x, y, \delta}\left|(n-1)^{-1} \sum_{i=1}^{n-1} \xi_{i}(x, y, \delta)-P\left(\xi_{1}(x, y, \delta)=1\right)\right| \stackrel{\text { a.s. }}{\rightarrow} 0,
$$

as $n \rightarrow \infty$. By Lipschitz continuity of $F$ we have $P\left(\xi_{i}(x, y, \delta)=1\right)=O(\eta)$ where the $O$ does not depend on $x, y, \delta$. Similarly, $\sup _{x, y, \delta}\left|(n-1)^{-1} \sum_{i=1}^{n-1} \zeta_{i}(x, y, \delta)\right| \stackrel{\text { a.s. }}{\rightarrow} O(\eta)$, as $n \rightarrow \infty$. Hence, we obtain

$$
\sup _{x, y, \delta}\left|\widehat{p}_{+n}(x, y ; \delta)-p_{+}(x, y ; \delta)\right| \stackrel{a . s .}{\longrightarrow} 0, \quad \sup _{x, y, \delta}\left|\widehat{p}_{-n}(x, y ; \delta)-p_{-}(x, y ; \delta)\right| \stackrel{a . s .}{\longrightarrow} 0,
$$

as $n \rightarrow \infty$. Note that this implies

$$
q\left(i, j, \widehat{p}_{+n}(x, y ; \delta), \widehat{p}_{-n}(x, y ; \delta)\right) \stackrel{a . s .}{\rightarrow} q\left(i, j, p_{+}(x, y ; \delta), p_{-}(x, y ; \delta)\right),
$$

uniformly in $(x, y, \delta)$ and $|i|<I$ and $|j|<K$, as $n \rightarrow \infty$. Consequently, the difference of the corresponding d.f.s converges uniformly in $(x, y, \delta)$ to 0 , i.e., for all $x^{\prime}, y^{\prime} \in \mathbb{R}$,

$$
\left.\mid \sum_{k \leq x^{\prime}} \sum_{l \leq y^{\prime}} q\left(k, l, \widehat{p}_{+n}(x, y ; \delta), \widehat{p}_{-n}(x, y ; \delta)\right)\right)-\sum_{k \leq x^{\prime}} \sum_{l \leq y^{\prime}} q\left(k, l, p_{+}(x, y ; \delta), p_{-}(x, y ; \delta)\right) \mid \rightarrow 0,
$$

as $n \rightarrow \infty$, w.p. 1, since there are only a finite number of summands. Noting that by definition $\widehat{P}_{1, L_{1}}, P_{1, L_{1}}, \widehat{P}_{2, \delta, L_{2}}$, and $P_{2 \delta, L_{2}}$ are finite sums, (19) follows.

\section{Simulations}

We conducted a simulation study to assess the accuracy of the proposed methods. In our first experiment i.i.d. $N(0,1)$ samples were generated, and the clipping median $\widehat{m}_{n}$ using the Gaussian kernel was calculated. For the simulation we used estimators without truncation, but the resulting procedure is very time consuming. Therefore, for time critical applications we recommend to use the proposed truncated estimators. Table 1 reports the simulated level of the test which rejects the null hypothesis that $\Delta^{\prime}=0$ if the estimated $p$ value is less than 0.025 or greater 0.975 . Each entry was obtained by 5,000 repetitions. It can be seen that the estimated $p$-values provide reliable statistical tests. 


\begin{tabular}{cccc}
\hline & \multicolumn{3}{c}{$h$} \\
$M$ & 25 & 50 & 100 \\
\hline 0.5 & 0.0360 & 0.0360 & 0.0466 \\
0.75 & 0.0372 & 0.0522 & 0.0554 \\
1 & 0.0488 & 0.0512 & 0.0494 \\
1.25 & 0.0418 & 0.0490 & 0.0526 \\
1.5 & 0.0428 & 0.0478 & 0.0446 \\
1.75 & 0.0352 & 0.0450 & 0.0448 \\
2 & 0.0244 & 0.0360 & 0.0468 \\
\hline
\end{tabular}

TABLE 1. Accuracy of the estimated null distribution for a two-sided significance test with nominal level $\alpha=0.05$

The second experiment deals with the more interesting mixture model. Test samples were simulated according to the model

$$
Y_{i} \sim \mathcal{N}(0,1), \quad i \in \mathcal{I}_{1}, \quad Y_{i} \sim \mathcal{N}(\Delta, 1), \quad i \in \mathcal{I}_{2},
$$

and $Y_{n} \sim \mathcal{N}\left(\Delta^{\prime}, 1\right)$. We used a Gaussian kernel (standard normal density) for $k$.

To mimick an object detection problem, we used $h=24,48$, and 63 , and $M=0.5,1,1.5$, and 2. The level shifts were chosen as $\Delta=1$ and $\Delta^{\prime}=0,1$. The number of background pixels $k$ was chosen to ensure $k / h=0.25$ and 0.5 . Since the computations are more time consuming in the mixture model case, each table entry was calculated based on 1,000 replications.

Table 2 provides estimates for the true level of the proposed procedure for a nominal level $\alpha=0.05$ using $\Delta^{\prime}=\Delta=1$, and power estimates for $\Delta^{\prime}=0$ and $\Delta=1$. It can be seen that the test procedure is conservative and also has power to detect small to moderate shifts. The results also indicate that there is an optimal value of $M$ which maximizes the power.

\section{ACKNOWLEDGEMENTS}

The work of the author was supported by the Deutsche Forschungsgemeinschaft, SFB 475 Reduction of Complexity in Multivariate Data Structures. 


\begin{tabular}{cccccc}
\hline $\begin{array}{c}\text { sample size } \\
h\end{array}$ & background & \multicolumn{5}{c}{$M$} \\
\hline Size $\left(\Delta^{\prime}=\Delta=1\right)$ & 0.5 & 1 & 1.5 & 2 \\
24 & 6 & 0.041 & 0.021 & 0.021 & 0.025 \\
& 12 & 0.036 & 0.024 & 0.019 & 0.015 \\
48 & 12 & 0.026 & 0.034 & 0.033 & 0.021 \\
& 24 & 0.024 & 0.017 & 0.028 & 0.018 \\
63 & 24 & 0.027 & 0.023 & 0.041 & 0.040 \\
& 48 & 0.024 & 0.028 & 0.032 & 0.023
\end{tabular}

Power for $\Delta^{\prime}=0, \Delta=1$.

\begin{tabular}{lccccc}
24 & 6 & 0.097 & 0.118 & 0.121 & 0.079 \\
& 12 & 0.090 & 0.115 & 0.109 & 0.071 \\
48 & 12 & 0.120 & 0.161 & 0.151 & 0.101 \\
& 24 & 0.126 & 0.161 & 0.144 & 0.106 \\
63 & 24 & 0.144 & 0.149 & 0.167 & 0.116 \\
& 48 & 0.129 & 0.157 & 0.165 & 0.116 \\
\hline
\end{tabular}

TABLE 2. Level and power of the test (16) to test the null hypothesis that the current pixel belongs to the object against the alternative that it belongs to the background for different sample sizes and parameter settings.

\section{REFERENCES}

[1] Blum J. (1955), On the convergence of empiric distributions. Ann. Math. Statist. 26, 527-529.

[2] Budinger T. et al. (1996), Mathematics and Physics of Emerging Biomedical Imaging. (National Academic Press, Washington D.C.)

[3] Chiu C. K., Glad I. K., Godtliebsen F., and Marron J. S. (1998), Edge-preserving smoothers for image processing, J. Amer. Statist. Assoc. 93, 526-556.

[4] DeHardt J. (1971), Generalizations of the Glivenko-Cantelli theorem. Ann. Math. Statist. 42, 2050-2055.

[5] Godtliebsen F. (1991), Noise reduction using Markov random fields, J. Magn. Reson. 92, 102-114. 
[6] Godtliebsen F. and Marron S. (1997), A nonlinear Gaussian filter applied to images with discontinuities. J. Nonparametr. Statist. 8, 21-43.

[7] Godtliebsen F. and Spjøtvoll E. (1991), Comparison of statistical methods in MR imaging, Int. J. Imag. Sys. Tech. 3, 33-39.

[8] Lee J. S. (1983), Digital image smoothing and the sigma filter. Comput. Vision Graph. 24, 255-269.

[9] Pawlak M., Rafajłowicz E., and Steland A. (2004), Detecting jumps in time series Nonparametric setting. J. Nonparam. Statist. 16, 329-347.

[10] Pawlak M. and Rafajłowicz E. (2000), Vertically weighted regression - a tool for nonlinear data analysis and constructing control charts. AStA 84, 367-388.

[11] Pawlak M. and Rafajłowicz E. (2001), Jump preserving signal reconstruction using vertical weighting. Nonlinear Anal-Theor. 47, 327-338.

[12] Shorack G.R. and Wellner J.A. (1986), Empirical Processes with Applications to Statistics, (Wiley, New York.)

[13] Steland A. (2002a), Nonparametric monitoring of financial time series by jumppreserving estimators. Stat. Pap. 43, 361-377.

[14] Steland A. (2004a), Jump-preserving monitoring of dependent time series under local alternatives. Statistics and Decision 21, 4, 343-366.

[15] Steland A. (2004b), Sequential control of time series by functionals of kernel-weighted empirical processes under local alternatives. Metrika, in press.

[16] WolframResearch (2004), Application documentation: Digital image processing package, Sec. 5.5 http://documents.wolfram.com/applications/digitalimage. 